\title{
Five POTE paralogs and their splice variants are expressed in human prostate and encode proteins of different lengths
}

\author{
Tapan K. Bera, Nancy Huynh, Hiroshi Maeda, Bangalore K. Sathyanarayana, \\ Byungkook Lee, Ira Pastan*
}

Laboratory of Molecular Biology, Center for Cancer Research, National Cancer Institute, National Institutes of Health, Building 37, MSC 4264. 37 Convent Drive Room 5106, Bethesda, MD 20892-4264, USA

Received 24 February 2004; accepted 6 May 2004

Available online 4 July 2004

Received by R. Di Lauro

\begin{abstract}
POTE is a new gene that contains ankyrin and spectrin domains and is expressed in prostate, testis, ovary, and placenta. In humans, 10 highly homologous variants of the gene are dispersed among eight chromosomes. POTE paralogs are detected in primates but not in other species. Using prostate RNA, we characterized cDNAs from five paralogs and their splice variants. The proteins encoded by the POTE paralogs and their variants range from 80 to $32 \mathrm{kDa}$. Transfection of POTE constructs into 293T cells shows that the POTE protein, like spectrin, is localized on the inner aspect of the plasma membrane. We also detect a noncoding transcript expressed on the opposite strand from POTE on chromosome 14 or 22. We speculate that POTE has an important signaling function in the reproductive system.
\end{abstract}

(C) 2004 Elsevier B.V. All rights reserved.

Keywords: Primate-specific gene; Membrane-associated protein; Ankyrin repeats; Spectrin motif; Noncoding RNA

\section{Introduction}

To identify new targets for the immunotherapy of prostate cancer, we have developed a computer-based screening strategy that generates clusters of ESTs that are expressed in prostate cancer but not in essential normal tissues (Vasmatzis et al., 1998). Using this approach, we have successfully identified several genes with this expression profile (Brinkmann et al., 1998; Essand et al., 1999; Wolfgang et al., 2000; Olsson et al., 2001; Bera et al., 2002a). One of these is a gene called POTE because it is expressed in prostate, ovary, testis, and placenta, as well as in prostate cancer (Bera et al., 2002b). The POTE gene has 11 exons and spans a 32$\mathrm{kb}$ region of human chromosome 21 . The $1.8-\mathrm{kb}$ transcript of POTE encodes a protein of molecular weight of $66 \mathrm{kDa}$. Amino acid sequence analysis of the POTE protein reveals that it has seven ankyrin repeats as well as spectrin motifs at

Abbreviations: CMV, cytomegalovirus; HA-tag, hemagglutinin-tag; PCR, polymerase chair reaction; PSNR, prostate-specific noncoding RNA; POTE, gene expressed in prostate, ovary, testis, and placenta.

* Corresponding author. Tel.: +301-496-4797; fax: +301-402-1344.

E-mail address: pastani@mail.nih.gov (I. Pastan). the carboxy-terminus of the protein. Ankyrin repeats are tandemly repeated modules of about 33 amino acids each, that are present in functionally diverse proteins and mediate protein-protein interactions (Michel et al., 2001). Spectrins, on the other hand, are major constituents of the cytoskeleton that are anchored to the plasma membrane by means of protein complexes that include ankyrins (Djinovic-Carugo et al., 2002). We have also reported that 10 paralogs of the POTE gene are dispersed among eight different chromosomes and at least five of them are expressed in prostate (Bera et al., 2002b). In this report, we describe the sequences of five POTE paralogs and their splice variants from prostate. The proteins encoded by the POTE paralogs and their splice variants vary in size suggesting their involvement in diverse regulatory pathways.

\section{Materials and methods}

\subsection{Primers}

Primers used in this study are T455, GGT AGA CGC GAT CTG TTC GCT ACT; T456, CCT AAG CTG TCC 


\section{ACT GTA CTT AAA; T474, GAC AGA CAC CAT CTG CTC GCT ACT A; T475, CTA AGC TGT CCA CTG TAC TGA ACT ACT G; T468, GTT GTC AAT GAC GAG CAC GGC GGT AT.}

\subsection{Cell lines}

PC-3 and 293T cells were acquired from the American Type Culture Collection (ATCC, Manassas, VA) and cultured in our laboratory according to the instructions from the supplier.

\subsection{Dot blot and northern blot hybridizations}

The human multiple tissue RNA dot blot (RNA Masterblot, Clontech, Palo Alto, CA) and Northern blot (Multiple Tissue Northern blot, Clontech) hybridizations were carried out as described previously (Bera et al., 2002b). Briefly, the RNA membranes were prehybridized for $2 \mathrm{~h}$ in hybridization solution (Hybrisol I, Oncor, Gaithersburg, MD) at $45{ }^{\circ} \mathrm{C}$. The probe was labeled with ${ }^{32} \mathrm{P}$ by random primer extension (Lofstrand Labs, Gaithersburg, MD), and incubated with the blots for $16 \mathrm{~h}$ at $45^{\circ} \mathrm{C}$. The blots were then washed $2 \times$ for 15 min each in $2 \times \mathrm{SSC}, 0.1 \% \mathrm{SDS}$, at room temperature and then washed $2 \times$ for $15 \mathrm{~min}$ each in $0.2 \times \mathrm{SSC}, 0.1 \% \mathrm{SDS}$, at $60{ }^{\circ} \mathrm{C}$. The membranes were then exposed to X-ray film for 1-2 days.

\subsection{Cloning and sequence analysis of POTE paralogs}

The POTE sequence was analyzed using the human genome browser (http://genome.cse.ucsc.edu/index. html org $=$ Human $\& d b=$ hg $12 \&$ hgsid $=21692782$ ). As we described earlier (Table 1) (Bera et al., 2002b), the POTE sequence aligned $99.8 \%$ to chromosome 21 . In addition, the POTE sequence aligned to several other chromosomes with significant homology. Based on the genomic sequence of each of the POTE paralogs, polymerase chain reaction (PCR) primers were designed. The full-length cDNA for each paralog was amplified from normal prostate cDNA by PCR using the corresponding gene specific primer pairs. The PCR product was gel purified and cloned into the pCR2.1 TOPO vector (Invitrogen, Carlsbad, CA). The clones were identified by restriction digestion and sequenced using Perkin-Elmer's rhodamine terminator sequencing kit (Perkin-Elmer Applied Systems, Warrington, UK).

\subsection{Expression vector construction}

The POTE-21 ORF lacking a termination codon was amplified by PCR and recombined into a Gateway Entry clone using pDonr223 (Invitrogen). Entry clones were sequence verified, and a mammalian expression clone was generated by recombining the entry clone with pDest-520, a

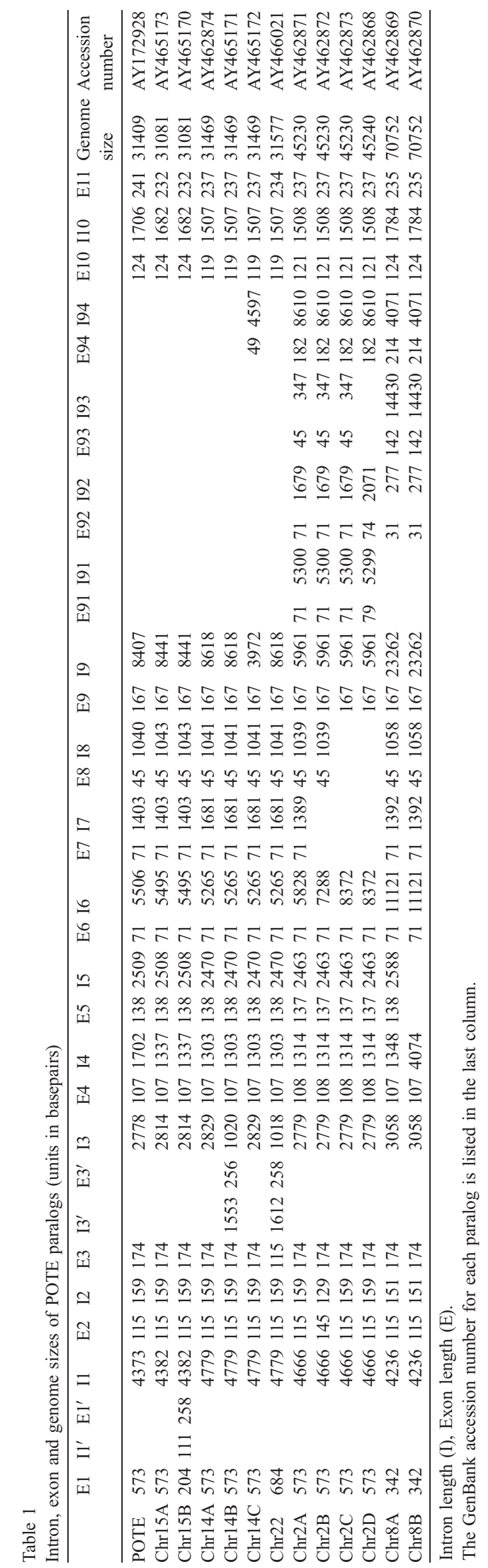


Gateway vector which creates a C-terminal hemagglutinintag (HA-tag) fusion under the control of the cytomegalovirus (CMV) promoter. Expression of this transcript leads to the production of a protein containing POTE protein fused to a 17-amino acid peptide containing the Gateway attB2 site and the HA-tag.

\subsection{In vitro transcription and coupled translation}

The in vitro transcription and translation of the POTE paralog cDNAs were carried out using T7 RNA polymerase and the rabbit reticulocyte lysate system (TNT; Promega, Madison, WI) following the manufacturer's instructions.

${ }^{35}$ S-Met (ICN, Costa Mesa, CA) was incorporated in the reaction for visualization of translated products. The reaction mixture was heated at $37{ }^{\circ} \mathrm{C}$ in reducing sample buffer containing $5 \mathrm{M}$ urea for $10 \mathrm{~min}$ and then analyzed under reducing conditions on a polyacrylamide gel (4-20\% PAGE, Tris/Glycine; Bio-Rad, Hercules, CA) together with a prestained protein molecular weight marker (Bio-Rad). The gel was dried and subjected to autoradiography.

\subsection{Immunocytochemistry and GFP observation}

PC-3 and 293T cells were cotransfected with pPOTE-HA and pEGFP-C1 (Clontech) plasmids. After a $48 \mathrm{~h}$ incubation following transfection of cells growing in a slide chamber, the cells were fixed for $10 \mathrm{~min}$ in $4 \%$ formaldehyde, treated for 5 min with $0.2 \%$ triton X-100 in PBS, blocked for 30 min with $0.4 \%$ normal goat globulin in PBS, and incubated at room temperature for $1 \mathrm{~h}$ with $5 \mu \mathrm{g} / \mathrm{ml}$ anti-HA-tag monoclonal antibodies (Roche Diagnostic, Indianapolis, IN). Subsequently, the cells were incubated at room tem- perature for $1 \mathrm{~h}$ with TRITC-conjugated secondary antibody (Jackson Laboratories, Bar Harbor, ME) then mounted in antifade solution with DAPI (Vector Laboratories, Burlingame, CA). Labeled cells were analyzed by laser confocal microscopy.

Transfection of pEGFP-C1 or POTE-GFP was done in PC-3 and 293T cells. Forty-eight hours after transfection, cells were fixed for $10 \mathrm{~min}$ in $4 \%$ formaldehyde, permeabilized with $0.1 \%$ Triton X100 in PBS at room temperature for $2 \mathrm{~min}$ and mounted in antifade solution with DAPI. GFP expression was observed by laser confocal microscopy.

\section{Results}

\subsection{Identification and cloning of full-length POTE paralogs from prostate}

\subsubsection{Cloning of the POTE paralog cDNA from chromo- some 15 (POTE-15)}

The original POTE cDNA was amplified from prostate RNA using PCR primers T455 and T456 from the gene located on chromosome 21q11.2 region (see Materials and methods; Fig. 1) (Bera et al., 2002b). During cloning, eight full-length cDNAs were isolated and sequenced. Four sequences aligned to chromosome 21 but the other four aligned to chromosome 15 with $100 \%$ identity, indicating that these four are transcripts from the chromosome 15 POTE paralog. Nucleotide sequence analysis reveals that there are two splice variants of the POTE paralog from chromosome 15. One variant, POTE-15A, has 11 exons like POTE (Fig. 1) and encodes a protein with 581 amino acids,
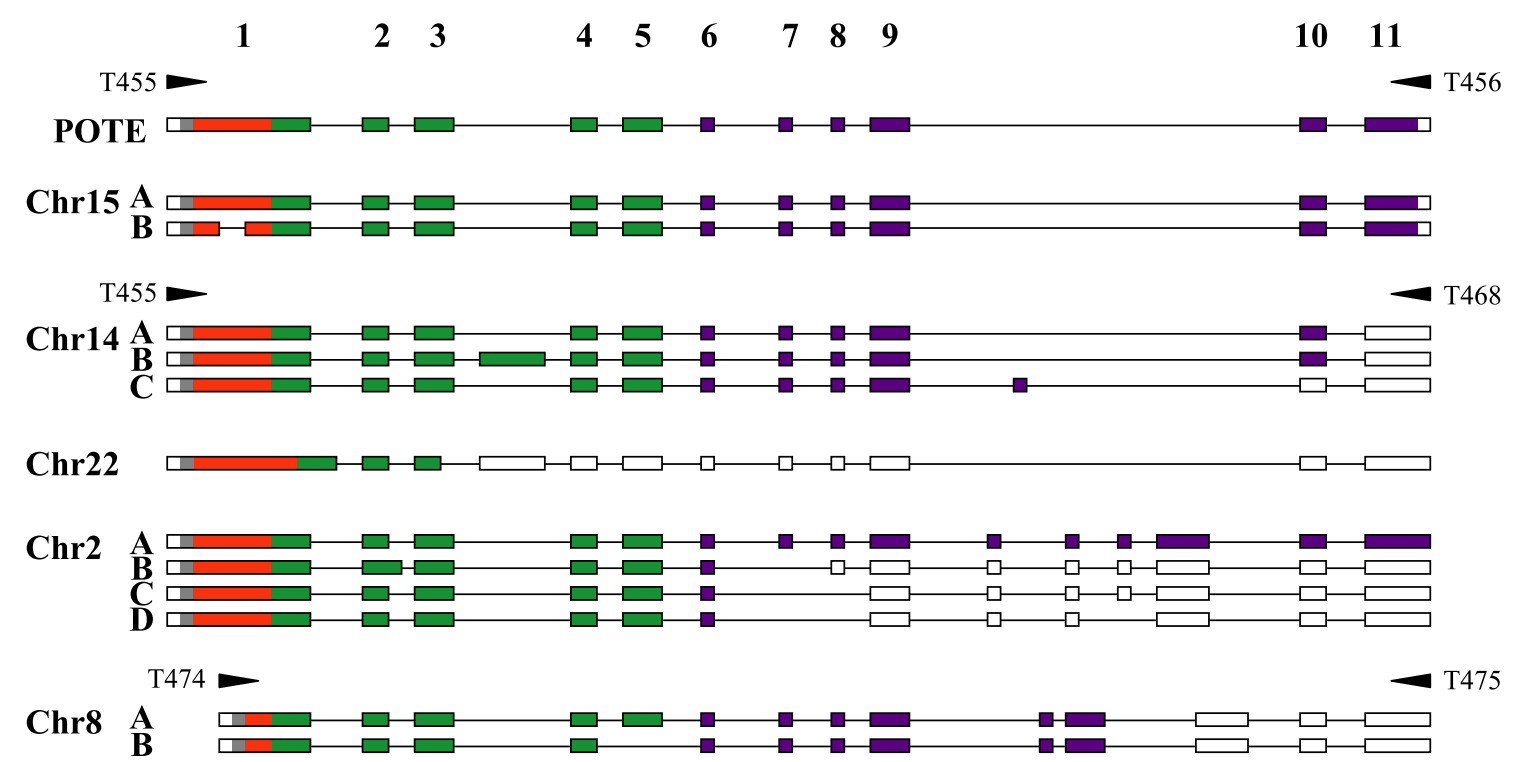

Fig. 1. Schematic showing the genomic organization of POTE paralogs and their splice variants. Square boxes represent exons and lines represent introns. Box sizes roughly correspond to relative exon sizes. The colors of the boxes indicate different regions of the proteins: red $=\mathrm{N}$-terminal repeat; green $=$ ankyrin repeats; and purple $=\mathrm{C}$-terminal helical domain. $3^{\prime \prime}$ and $5^{\prime \prime}$ UTR are not in color. 
three amino acids shorter than POTE (Fig. 2). All POTE$15 \mathrm{~A}$ exons are similar in size to those of POTE except that POTE-15A has a deletion of nine bases in the last exon. As shown in Table 1, the sizes of the introns of POTE-15A are similar to those of POTE but there are small differences in the size of most of the introns. The amino acid sequence analysis of the predicted ORF for POTE-15A, shows 97\% sequence identity with POTE. Both have three cysteine-rich repeats of 37 amino acids each at the amino-terminus, six or seven ankyrin repeats in the middle and a spectrin-like helical region at the carboxy-terminus (Fig. 2).

3.1.1.1. Splice variant of chromosome 15 paralog. One of the four cDNAs aligning to the chromosome 15 paralog is somewhat shorter and called POTE-15B (Fig. 1). POTE$15 \mathrm{~B}$ is $1706 \mathrm{bp}$ in size, which is $111 \mathrm{bp}$ shorter than POTE15A. Nucleotide sequence analysis of POTE-15B reveals that exons $2-11$ are identical to those in POTE-15A but exon 1 is $111 \mathrm{bp}$ shorter (Table 1). The cDNA of this variant encodes a protein containing 544 amino acids and lacks one of the three cysteine-rich repeats at the Nterminus (Fig. 2).

3.1.2. Cloning of the POTE paralog cDNAs from chromosomes 2, 14 and 22 (POTE-2, POTE-14 and POTE-22)

Exon 11 of the POTE gene aligns to the paralogs on chromosomes 2, 14 and 22 but with poor homology. To amplify the full-length POTE paralogs from chromosomes 2, 14 and 22, a $3^{\prime}$ primer T468 was synthesized and primers T455 and T468 were used for the PCR reaction utilizing prostate cDNA as the template (Materials and methods; Fig. 1). The PCR products were cloned into a TA-TOPO vector and sequenced. We have analyzed 40 clones, which have the correct size insert. After the sequencing of these clones, we are able to identify cDNAs that correspond to the transcript from the paralogs on chromosomes 2, 14 and 22.

3.1.2.1. POTE-14 and its splice variants. As shown in Fig. 1, we have identified three types of cDNA clones which align to chromosome 14 with $99.8 \%$ identity. POTE14A, like POTE, has 11 exons (Fig. 2). The sizes of the exons 1-9 of POTE-14A are the same as POTE but exon 10 is five bases shorter (Table 1). This causes the ORF to be terminated in exon 10 and generates a protein of 508 amino acids. The intron sizes are more or less similar to those of POTE.

POTE-14B and -14C are splice variants of POTE-14A. POTE-14B contains an additional exon of 255 bases between exons 3 and 4 as compared to POTE-14A (Fig. 1; Table 1). POTE-14C has an additional exon of 49 bases between exons 9 and 10. The predicted ORF for POTE-14B is 594-amino acids in length, and for POTE-14C because of a premature stop codon within the 49-bp additional exon, it is 487 amino acids long (Fig. 2).
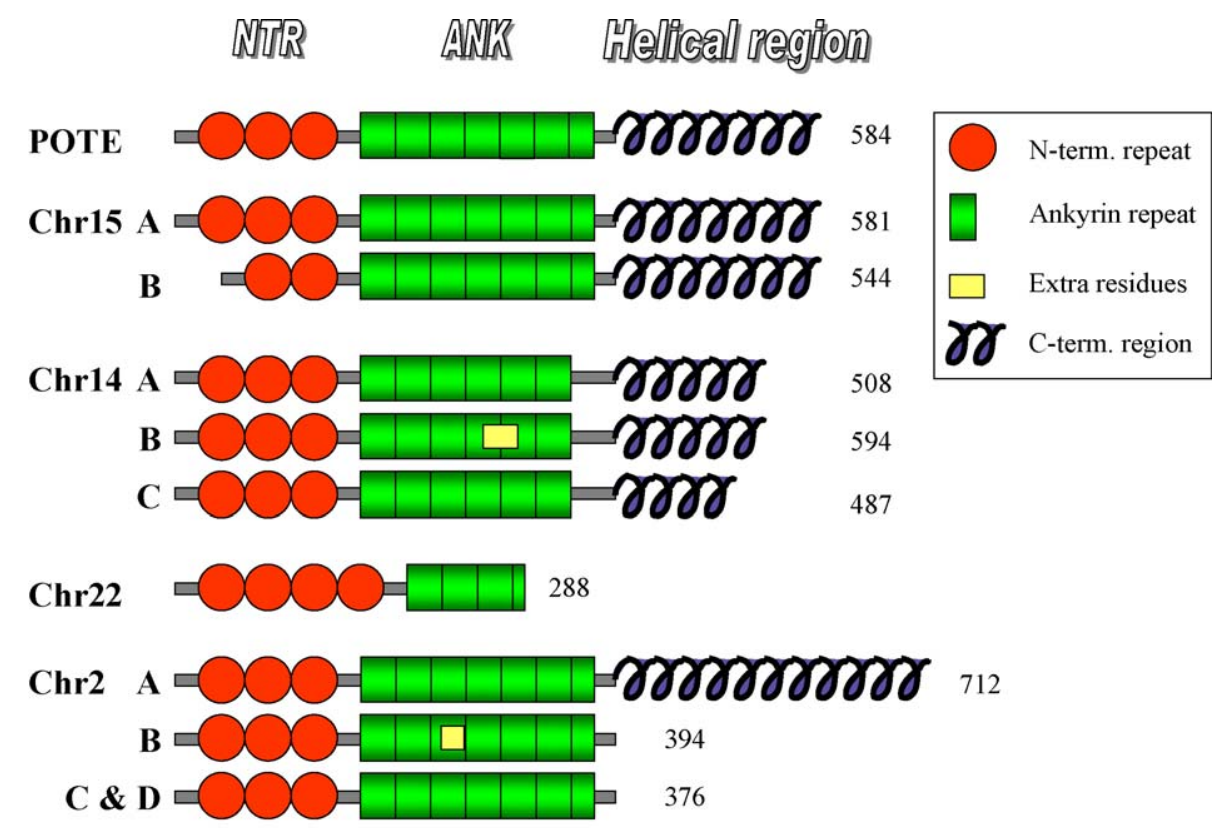

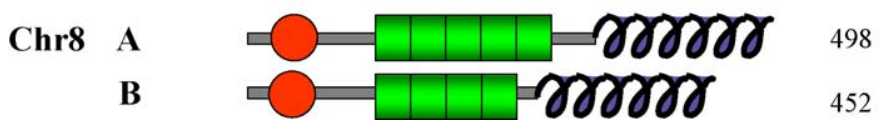

Fig. 2. Schematics describing the proteins encoded by POTE paralogs and their splice variants. Circles represent the 17-amino acid amino-terminal repeats, square boxes represent the ankyrin repeats and curved lines represent the carboxy-terminal domain, which includes the spectrin-like structure. 
3.1.2.2. POTE-22. The POTE-14 paralog sequences also aligned to chromosome 22 with similar identity to their alignment with chromosome 14 making it difficult to determine which cDNA corresponds to which chromosome. However, we have identified a group of cDNAs, which have a different exon 1 sequence from the POTE-14 paralogs. As shown in Fig. 1 and Table 1, the genomic organization of POTE paralog 22 is very similar to POTE-14B except for exons 1 and 3. There is an additional 111-bp sequence in exon 1 for POTE-22 cDNA, which is absent in POTE-14 or its splice variants. Exon 3 on the other hand is $59 \mathrm{bp}$ shorter (Table 1). Because of these differences, the POTE-22 cDNA encodes a predicted protein of 288 amino acids in length. As shown in Fig. 2, the predicted protein is missing the last three ankyrin repeats and part of the fourth. In addition, because of the additional 111-bp sequences in the first exon, this protein will have four cysteine-rich repeats at the amino-terminal end.

3.1.2.3. POTE-2 and its splice variants. We have identified four different types of cDNA clones that align to chromosome 2 with 99.8\% identity. As shown in Fig. 1, POTE-2A contains 11 exons like POTE, but it has an additional four exons within intron 9. The predicted ORF for POTE-2A cDNA encodes a protein with 712 amino acids (Fig. 2). POTE-2B, $-2 \mathrm{C}$ and $-2 \mathrm{D}$ are splice variants of POTE-2A. POTE-2B and $-2 \mathrm{C}$ are very similar to POTE-2A but POTE-2B lacks exon 7 and POTE-2C lacks exons 7 and 8. In addition, the second exon of POTE-2B is 30 nucleotides longer than other POTE paralogs and their splice variants (Table 1). POTE-2D on the other hand is very similar to POTE-2C, but lacks exon E9-3 (Table 1). The predicted ORFs for POTE-2B, $-2 \mathrm{C}$ and $-2 \mathrm{D}$ encode proteins that are 394, 376 and 376-amino acids long, respectively (Fig. 2). The POTE-2 paralog gene is spread across a $45-\mathrm{kb}$ region of chromosome 2 and is considerable longer than the 31-kb region of POTE, POTE-14, POTE-15 and POTE-22 (Table 1).

\subsubsection{Cloning of the POTE paralog cDNA from chromo- some 8 (POTE-8)}

Based on the sequence alignment of POTE to chromosome 8, oligos T474 and T475 (Fig. 1) were synthesized to identify the sequences of the POTE paralogs from chromosome 8 . These primers were used in a PCR reaction to generate cDNA to correspond to POTE-8 paralogs utilizing prostate cDNA as the template. The PCR product was then cloned and sequenced. We have analyzed 24 clones and as shown in Fig. 1, two different types of cDNAs, POTE-8A and POTE-8B, have been identified with a $99.9 \%$ match to chromosome 8 . Nucleotide sequence analysis of POTE-8A reveals that it has 14 exons, 11 of which correspond to those of POTE. However, the first exon is only 342 nucleotides in length. The three extra exons are between E9 and E10 (Table 1). POTE-8B is a splice variant of POTE-8A and is missing exon 5 . The predicted ORF for POTE-8A and $-8 \mathrm{~B}$ encode proteins that are 498 and 452 amino acids in length, respectively (Fig. 2). The POTE-8 paralog gene occupies a $71 \mathrm{~kb}$ region of chromosome 8 and is the largest of the paralog members (Table 1).
A

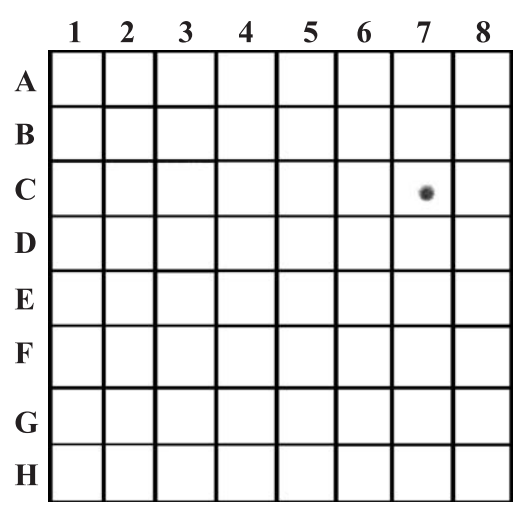

\section{C}

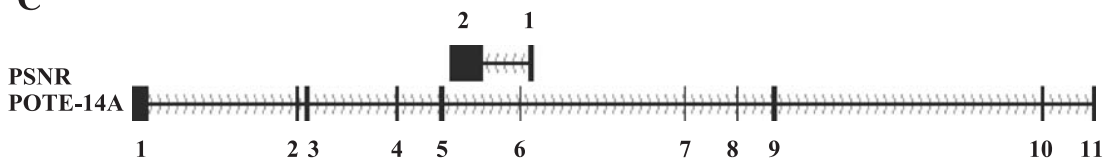

B

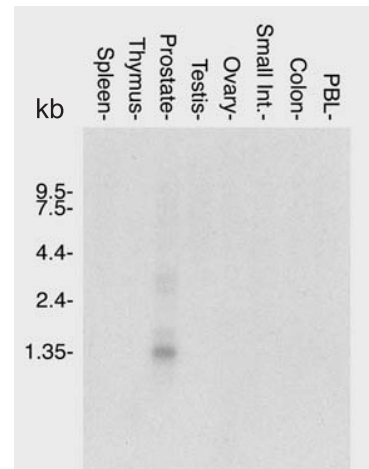

Fig. 3. Genomic organization and tissue distribution of PSNR mRNA. (A) RNA hybridization of a multiple tissue dot blot containing mRNA from 50 normal human cell types or tissues using a cDNA probe of the PSNR transcript. Strong expression is observed in prostate (C7), but no detectable expression in brain (A1), heart (C1), kidney (E1), liver (E2), lung (F2) and adrenal gland (D5). (B) Northern blot analysis showing expression and transcript sizes of PSNR in different normal tissues. The predominant transcript is about $1.25 \mathrm{~kb}$ in size and is expressed only in prostate tissue. (C) Schematics showing the genomic organization of PSNR and POTE-14A. Numbers represent the exon number for each gene. There are 11 exons for POTE-14A and two exons for PSNR. 


\subsection{New prostate-specific gene in the POTE locus}

We previously reported the presence of a new prostatespecific transcript expressed in the POTE locus but from the opposite strand (Bera et al., 2002b). Analysis of the EST database shows that there are 15 ESTs derived from this transcript of which 12 are from normal prostate, two from prostate cancer and one from normal kidney. To experimentally investigate the expression of this transcript we performed a mRNA dot blot (human RNA master blot, Clontech) using a PCR-generated probe from the consensus EST sequence of this cluster. As shown in Fig. $3 \mathrm{~A}$, a strong signal was detected only in prostate tissue mRNA (position C7) and not in kidney, indicating a restricted expression of this transcript to prostate tissue. To determine the size of the transcript we probed a multiple tissue northern blot (Clontech). As shown in Fig. $3 \mathrm{~B}$, the mRNA is only detected in the prostate lane; the transcript is $1.25 \mathrm{~kb}$ in size. To isolate the full-length cDNA we performed a $5^{\prime}$ and $3^{\prime}$ RACE-PCR using normal prostate cDNA (Marathon-Ready cDNA, Clontech). The RACE-PCR performed at the $5^{\prime}$ end give rise to a cDNA of $1.25 \mathrm{~kb}$ in size. We were not able to get additional bases at the $3^{\prime}$ end by performing $3^{\prime}$ RACEPCR indicating that the major transcript ends at this position. The nucleotide sequence analysis of the 1.25$\mathrm{kb}$ cDNA (GenBank accession number AY458019) reveals that it contains many stop codons suggesting the cDNA is noncoding. We have named the gene prostatespecific noncoding RNA (PSNR).

Sequence alignment of the PSNR cDNA with the human genome sequence shows $100 \%$ match to chromosome 14 and a $99.9 \%$ match to chromosome 22 at the POTE locus but from the opposite direction (Fig. 3C). In addition, as shown in Table 2, the PSNR sequence aligns to seven additional loci on human chromosomes. However, analysis of 20 independent cDNA sequences from prostate reveals that PSNR is only transcribed from chromosomes 22 and 14. It is not established whether the transcript arises from both chromosomes, or only one.

Table 2

Alignment of the PSNR sequence at different chromosomal locations

\begin{tabular}{|c|c|c|c|c|c|c|c|}
\hline \multirow[t]{2}{*}{ Chro } & \multirow[t]{2}{*}{ Strand } & \multicolumn{2}{|c|}{ Chromosome } & \multirow[t]{2}{*}{ Score } & \multicolumn{2}{|c|}{ cDNA } & \multirow{2}{*}{$\begin{array}{l}\text { Identity } \\
(\%)\end{array}$} \\
\hline & & Start & End & & Start & End & \\
\hline 14 & + & 17378241 & 17380976 & 1256 & 1 & 1259 & 100.0 \\
\hline 22 & + & 13224829 & 13227564 & 1254 & 1 & 1259 & 99.9 \\
\hline 2 & + & 128166168 & 128499340 & 1105 & 1 & 1259 & 94.6 \\
\hline 2 & - & 128622144 & 128624868 & 1103 & 1 & 1259 & 94.5 \\
\hline 2 & - & 129915021 & 129917747 & 1091 & 1 & 1259 & 94.2 \\
\hline 2 & + & 128151596 & 128168893 & 1081 & 1 & 1259 & 93.8 \\
\hline 21 & - & 11654671 & 11657445 & 1065 & 1 & 1259 & 94.0 \\
\hline 15 & - & 18733018 & 18735791 & 1030 & 1 & 1259 & 93.0 \\
\hline 8 & - & 42890563 & 42893427 & 862 & 1 & 1259 & 88.5 \\
\hline
\end{tabular}

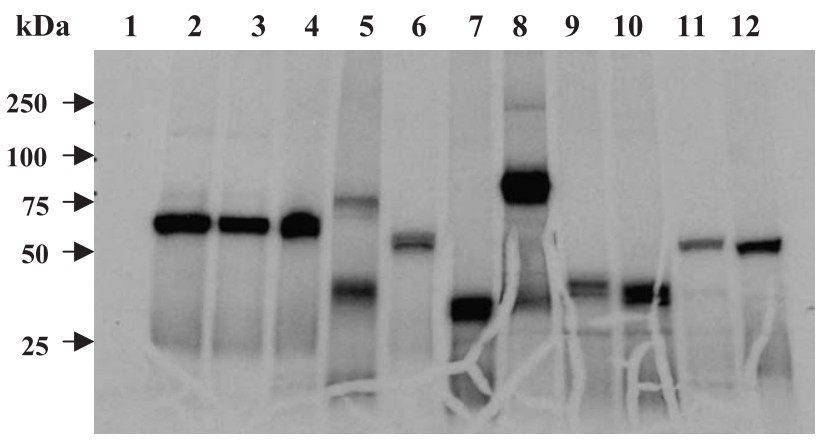

Fig. 4. Analysis of the protein encoded by POTE paralogs and their splice variants. Different cDNA were transcribed in vitro with T7 RNA polymerase and the RNA was translated with rabbit reticulocyte lysate (TNT system) in the presence of ${ }^{35} S$-methionine. The translated products were analyzed by SDS-PAGE and fluorography. Lanes are (1) empty vector control, (2) POTE-15A, (3) POTE-15B, (4) POTE-14A, (5) POTE-14B, (6) POTE-14C, (7) POTE-22, (8) POTE-2A, (9) POTE-2B, (10) POTE-2C, (11) POTE-8A, and (12) POTE-8B.

\subsection{In vitro transcription and translation of the POTE paralogs and their splice variant $c D N A S$}

The POTE paralog cDNAs and their splice variants isolated from prostate have predicted ORFs of many different sizes (Fig. 2). To determine the size of the proteins encoded by these cDNAs, in vitro transcription and translation was performed. SDS-PAGE analysis of the translated products (Fig. 4) show that the POTE paralog cDNAs and their splice variants encode protein products that are in agreement with the predicted ORFs of the cDNA. The predicted and observed sizes of the protein products of POTE paralogs and their splice variants are summarized in Table 3. The calculated molecular mass for POTE-15A and -15B are 65.8 and $61.1 \mathrm{kDa}$, respectively. The sizes of the in vitro translated products for these cDNAs agree with the calculated mass (Fig. 4, lanes 2 and 3; Table 3). The calculated molecular mass for POTE$14 \mathrm{~A},-14 \mathrm{~B}$ and $-14 \mathrm{C}$ are $57.2,67.1$ and $54.6 \mathrm{kDa}$, respectively, which matches with the sizes of the in vitro translated products for these cDNAs (Fig. 4, lanes 4, 5

Table 3

Predicted and observed sizes of the protein products of POTE paralogs and their splice variants

\begin{tabular}{lll}
\hline cDNA & Predicted size in $\mathrm{kDa}$ & Observed size in $\mathrm{kDa}$ \\
\hline POTE-15A & 65.8 & 65 \\
POTE-15B & 61.1 & 62 \\
POTE-14A & 57.2 & 58 \\
POTE-14B & 67.1 & 71 \\
POTE-14C & 54.6 & 55 \\
POTE-22 & 32.3 & 34 \\
POTE-2A & 72.5 & 75 \\
POTE-2B & 40.2 & 40 \\
POTE-2C & 38.6 & 39 \\
POTE-8A & 56.2 & 56 \\
POTE-8B & 51.3 & 52 \\
\hline
\end{tabular}


and 6; Table 3). The in vitro translated product for the POTE-22 agrees with the predicted molecular mass of 32.3 kDa (Fig. 4, lane 7; Table 3). As shown in Fig. 4 (lanes 810) the in vitro translated products for POTE-2A, $-2 B$ and $-2 \mathrm{C}$ are 75,40 and $39 \mathrm{kDa}$ in size, respectively, which agree with the predicted molecular masses. Similarly, the calculated molecular mass for POTE-8A and $-8 \mathrm{~B}$ are 56.2 and $51.3 \mathrm{kDa}$, which agrees with the sizes of the in vitro translated products for their corresponding cDNAs (Fig. 4, lanes 11 and 12; Table 3).

\subsection{POTE protein is associated with the plasma membrane}

The POTE protein has a spectrin-like structure at the carboxy-terminus preceded by six or seven ankyrin repeats. Because spectrin is associated with the inner surface of the plasma membrane, it seemed possible that POTE might have a similar location in the cell. To determine the location of the protein in the cell, we transfected PC-3 cells with an expression plasmid encoding POTE with a HA epitope tag at the carboxy-terminus and determined the location of the protein by immunofluorescence with an anti-HA-tag antibody. As shown in Fig. 5A, a strong signal was detected around the periphery of the cell. In addition, there were some punctate signals in the cytoplasm, presumably due to aggregation of overexpressed protein. We obtained similar results in $293 \mathrm{~T}$ cells (data not shown). In another experiment we introduced a POTE-EGFP construct into PC-3 cells and visualized the fluorescent pattern in cells. As shown in Fig. 5B, positive signals were found mainly along the plasma membrane with some scattered punctate reac- tivity in the cytoplasm. In contrast, EGFP alone is detected throughout the cytoplasm. These results suggest that, like spectrin, POTE protein is mainly associated with the plasma membrane of the cell (Djinovic-Carugo et al., 2002). Because POTE does not have a signal sequence we assume it is located on the inner aspect of the cell membrane, but this assumption remains to be confirmed experimentally.

\section{Discussion}

Previously, we described a new gene, POTE, which has many paralogs in the human genome and other primate genomes (Bera et al., 2002b). There is no evidence of the presence of any orthologs in the mouse or the rat. The fact that the POTE gene family has only been found in primates, and that there are many paralogs located on eight different chromosomes in humans, indicate that POTE has one or more special functions. The finding that POTE is only expressed in testis, prostate, ovary and placenta indicates an important role in primate reproduction or speciation. Another unusual feature of POTE is that it contains ankyrinand spectrin-like domains, as well as a new type of cysteinerich repeats at its amino-terminus.

In this report, we describe the cloning and sequencing of cDNAs derived from five POTE paralogs that are expressed in normal prostate. We find that there are many splice variants of POTE paralogs encoding proteins of vastly different sizes and affecting the composition of all three major domains.
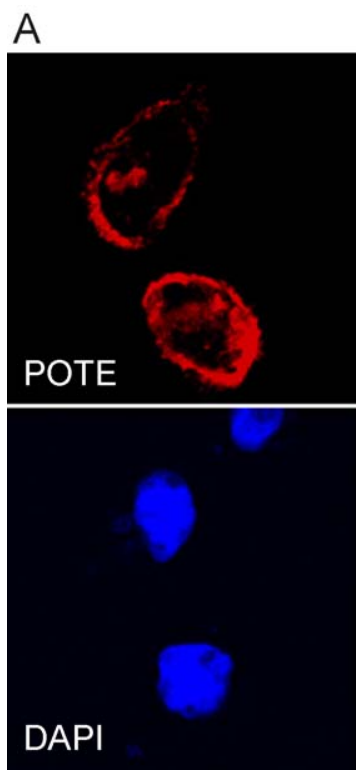
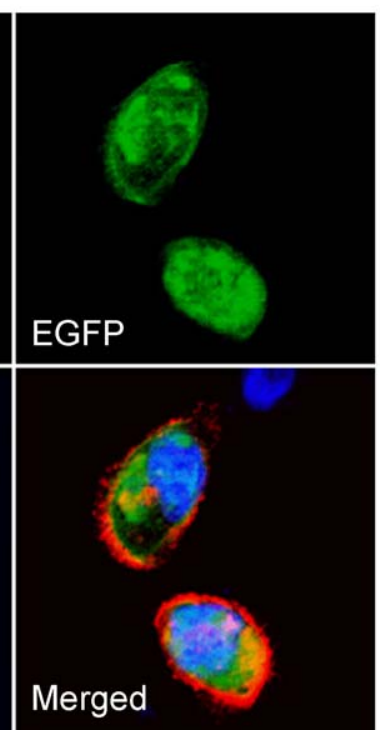

B

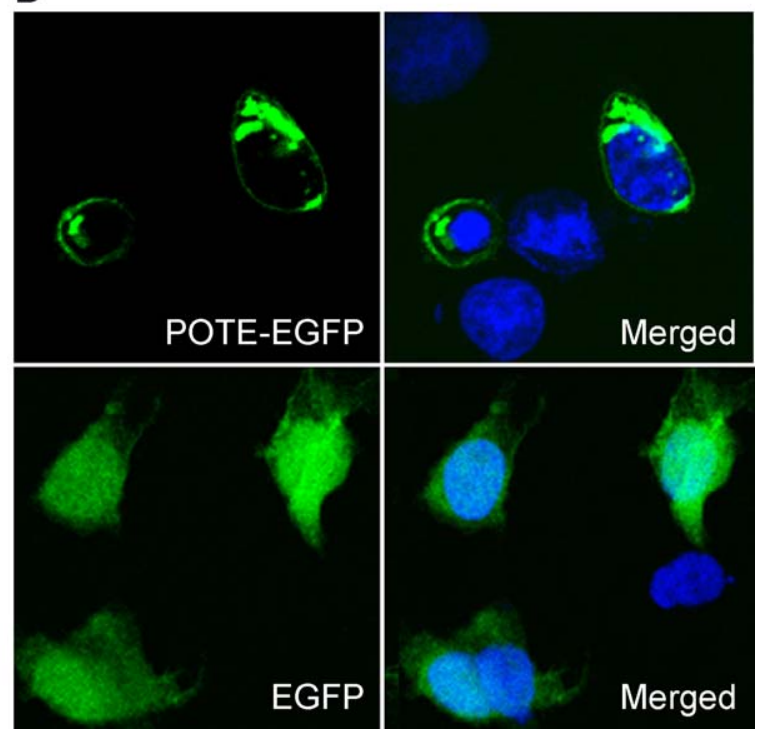

Fig. 5. Cellular localization of POTE protein. (A) Prostate cancer cell line, PC-3, was transiently transfected with an expression plasmid containing POTE with a HA tag at the carboxy-terminus. Cells were labeled with anti-HA primary antibody followed by TRITC-conjugated secondary antibody and visualized under a confocal microscope. The red signals representing POTE expression were localized mainly in the plasma membrane of PC-3 cells. (B) PC-3 cells were transiently transfected with either POTE-EGP construct (upper panel) or EGFP alone (lower panel) and visualized under a confocal microscope after fixation with formaldehyde. After permeabilization, nuclei were stained with DAPI (blue). Right panels are merged images with EGFP (green) and DAPI (blue) signals. 


\subsection{Genome size of POTE paralogs is different}

The POTE gene contains 11 exons and spans over 31.4$\mathrm{kb}$ regions of the genome. The genome sizes for POTE-15, POTE-14 and POTE-22 paralogs are very similar to that of POTE, but the genome size of the POTE- 8 paralog is 70.7 $\mathrm{kb}$, a little over twice the size of POTE. However, the exon sizes (exons 2-11) between POTE and that of POTE- 8 are very similar. Essentially, all of the increase in the size of the gene is due to the expansion of introns 6 , 9, and 9-3. The genome size of the POTE-2 paralog is $45.2 \mathrm{~kb}$, in between the sizes of POTE and POTE-8. Like POTE- 8 , the differences are mostly in the intronic regions of the gene.

\subsection{Splice variants of POTE paralogs encode different size protein and may have different functions}

POTE encodes a 66-kDa protein with six or seven ankyrin repeats in the middle of the molecule, a spectrinlike structure at the carboxy-terminus and three cysteine rich repeats of 17 amino acids each at the amino-terminus. Most of its paralogs encode proteins with similar types of motifs. However, most of the paralogs have splice variants capable of encoding proteins of different sizes as well as proteins with different motif structures. For example, POTE-15B lacks one of the three repeats at the aminoterminus of the protein. POTE-14C lacks the spectrin-like feature at the carboxy-terminus. Paralog POTE-22 lacks not only the C-terminal helical structure, but also three of the ankyrin repeats and has four instead of three cysteinerich repeats at the amino-terminus. Sequence analysis suggests that POTE-14B may contain a membrane-spanning region between ankyrin repeats 4 and 5 that needs to be confirmed experimentally. Although the functional significance of the amino-terminal cysteine-rich repeats of POTE is unknown, the functions of ankyrin motif and the spectrin protein have been studied in detail (Michel et al., 2001; Djinovic-Carugo et al., 2002). Ankyrin repeats have been identified in numerous functionally diverse proteins and are involved in protein-protein interactions in many functional pathways within the cell. Spectrins on the other hand are known to be the main constituents of the cytoskeleton, which is anchored to the plasma membrane by means of protein complexes that include ankyrins. In POTE, spectrin and ankyrin motifs are present in one molecule. It is probable that the protein products from these POTE paralogs and their splice variants are involved in different signaling pathways and some domain might have adaptor functions. Our immunofluresence experiments show that POTE, like spectrin, appears to be localized to the plasma membrane, suggesting the possibility that POTE might be involved in transmitting signals from the cell surface. We plan to investigate the subcellular localization of other paralog proteins as well as that of their splice variants.

\subsection{PSNR may regulate the expression of POTE paralogs in prostate}

PSNR is the third member of a group of recently described prostate-specific genes that transcribe noncoding RNAs (Bussemakers et al., 1999; Srikantan et al., 2000). Like DD3 and PCGEM, the function of PSNR remains to be determined. However, the location of the $P S N R$ gene within the POTE locus and its transcription from the strand opposite to that of the POTE gene suggests that PSNR may have a regulatory role in POTE function in prostate.

In this study, we have only characterized the POTE paralogs expressed in normal prostate and found that five of the POTE paralogs are expressed. We have begun to analyze POTE paralogs expressed in prostate cancer and find that only some of these paralogs are expressed (unpublished data). We have not yet detected expression in prostate of POTE paralogs located on chromosomes 13 and 18, but these may be expressed in testis, ovary or placenta, other tissues in which POTE has been detected (Bera et al., 2002b).

In summary, we have characterized the POTE paralogs expressed in normal prostate. Transcripts from chromosomes $2,8,14,15$ and 22 were detected, including several different spliced forms. The proteins encoded by these RNAs range from 32 to $80 \mathrm{kDa}$ in size, suggesting POTE has a variety of cellular functions.

\section{Acknowledgements}

We thank members of the Pastan laboratory for reviewing the manuscript, Susan Garfield and Stephen Wincovitch, CCR Confocal Microscopy Core Facility, for technical assistance in confocal microscopy, Maria Gallo for reading of the manuscript, and Anna Mazzuca for editorial assistance.

\section{References}

Bera, T.K., Maitra, R., Iavarone, C., Salvatore, G., Kumar, V., Vincent, J.J., Sathyanarayana, B.K., Duray, P., Lee, B.K., Pastan, I., 2002a. Discovery of three genes specifically expressed in human prostate by expressed sequence tag database analysis. Proc. Natl. Acad. Sci. U. S. A. 99, 3058-3063.

Bera, T.K., Zimonjic, D.B., Popescu, N.C., Sathyanarayana, B.K., Kumar, V., Lee, B., Pastan, I., 2002b. POTE, a highly homologous gene family located on numerous chromosomes and expressed in prostate, ovary, testis, placenta and prostate cancer. Proc. Natl. Acad. Sci. U. S. A. 99, 16975-16980.

Brinkmann, U., Vasmatzis, G., Lee, B., Yerushalmi, N., Essand, M., Pastan, I., 1998. PAGE-1, an X chromosome-linked GAGE-like gene that is expressed in normal and neoplastic prostate, testis and uterus. Proc. Natl. Acad. Sci. U. S. A. 95, 10757-10762.

Bussemakers, M.J., van Bokhoven, A., Verhaegh, G.W., Smit, F.P., Karthaus, H.F., Schalken, J.A., Debruyne, F.M., Ru, N., Isaacs, W.B., 1999. DD3: a new prostate-specific gene, highly overexpressed in prostate cancer. Cancer Res. 59, 5975-5979. 
Djinovic-Carugo, K., Gautel, M., Ylanne, J., Young, P., 2002. The spectrin repeat: a structural platform for cytoskeletal protein assemblies. FEBS Lett. 513, 119-123.

Essand, M., Vasmatzis, G., Brinkmann, U., Duray, P., Lee, B., Pastan, I., 1999. High expression of a specific T-cell receptor gamma transcript in epithelian cells of the prostate. Proc. Natl. Acad. Sci. U. S. A. 96, 9287-9292.

Michel, F., Solter-Lopex, M., Petosa, C., Cramer, P., Siebenlist, U., Müller, W., 2001. Crystal structure of the ankyrin repeat domain of Bcl-3: a unique member of the IKB protein family. EMBO J. 20, 6180-6190.

Olsson, P., Bera, T.K., Essand, M., Kumar, V., Duray, P., Vincent, J., Lee, B.K., Pastan, I., 2001. GDEP, a new gene differentially expressed in normal prostate and prostate cancer. Prostate 48, 231-241.
Srikantan, V., Zou, Z., Petrovics, G., Xu, L., Augustus, M., Davis, L., Livezey, J.R., Connell, T., Sesterhenn, I.A., Yoshino, K., Buzard, G.S., Mostofi, F.K., McLeod, D.G., Moul, J.W., Srivastava, S., 2000. PCGEM1, a prostate-specific gene, is overexpressed in prostate cancer. Proc. Natl. Acad. Sci. U. S. A. 97, 12216-12221.

Vasmatzis, G., Essand, M., Brinkmann, U., Lee, B., Pastan, I., 1998. Discovery of three genes specifically expressed in human prostate by expressed sequence tag database analysis. Proc. Natl. Acad. Sci. U. S. A. $95,300-304$.

Wolfgang, C.W., Essand, M., Vincent, J.J., Lee, B., Pastan, I., 2000. TARP: a nuclear protein expressed in prostate and breast cancer cells derived from an alternate reading frame of the $\mathrm{T}$ cell receptor $\gamma$ chain locus. Proc. Natl. Acad. Sci. U. S. A. 97, 9437-9442. 